\title{
Wind Power Real-time Prediction Based on Improved Time Series and Grey Model
}

\author{
Lanlan Chen ${ }^{1, a^{*}}$, Zlxia Pei ${ }^{1, b}$,Anjia Mao ${ }^{1, c}$, Yan LIU ${ }^{2, d}$, Zhuohang WU ${ }^{2, e}$, \\ and Yuzheng $\mathrm{HOU}^{2, \mathrm{f}}$ \\ ${ }^{1}$ No.2,Rd.Beinong, Changping District, Beijing, China \\ ${ }^{2}$ No.183,Rd.Wencui,Shenyang, China

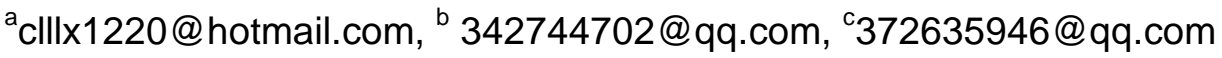

Keywords: wind power, time series, grey model, combination model

Abstract. This paper aims to establish a suitable wind power forecasting model used to forecast the power of the wind farm. An improved real-time series model is built by linear function and Fourier function. For raw data, the picture of historical data is adopted to correct them. In order to improve the prediction accuracy of wind power, it proposes the linear combination model based on improved time series model and grey model. The model uses a fixed weight method. Mathematical analysis and calculation results show that combination model, which has certain reference value, is simple and can improve the prediction accuracy overall.

\section{Introduction}

When large scale wind farms are connected to the power grid, the power fluctuation of wind power will have a negative effect on the power balance and frequency regulation [1]. It is conducive to the safety and stable operation of power system to predict wind power more accurate.

According to the requirements of dispatch department, wind power recently forecast aims at the power value of the next day at 0:00 to $24: 00 \mathrm{pm}$, while real-time prediction is rolling forecast 16 point within future four hours from the specified point, and both the time resolution are $15 \mathrm{~min}$. Many model has been used for wind power prediction, the physical method requires the use of numerical weather prediction. Statistical methods are based on statistical analysis of historical data, then select the appropriate statistical model for predicting, among which the time series is the most typical method. Learning methods mainly refers to the use of artificial intelligence such as genetic algorithm, BP neural network and particle swarm optimization method to predict the future power value. Compared with statistical method, the calculation of learning model is larger but prediction results are more accurate [2].

Improving the accuracy of wind power forecasting in real time is of great significance to improve the operation performance of the wind power network. The combination forecasting method is one of the important development directions of the wind power prediction. [3] establishes a linear combination model of time series model and RBF neural network model, based on single forecasting model. [4] proposes a wind power prediction model based on integration of principal component analysis with back-propagation neural network. In the reference [5], principal component analysis is applied to process original input data, the principal components are used as input data for neural network. Studies have shown that different forecasting methods contain different information, and the combination of single method can improve the prediction accuracy.

In this paper, a combined model based on improved time series and grey prediction is proposed, which focuses on the research of real-time rolling forecast of wind power. Fristly, by use of linear function and Fourier function, it creates an improved time series model. Then, a method of data correction based on the picture of historical data to determine the range of time series is proposed. Finally, apply the improved time series, grey prediction and combination model to instances in turn, discussing the effectiveness and accuracy of models. 


\section{Combined model based on improved time series and grey prediction}

Improved time series model. In consideration of the accuracy and simplicity of calculation, to establish the real time series model based on linear function and Fourier function.

$$
\left\{\begin{array}{l}
Y_{i}(t)=f_{i}(t)+p_{i}(t) \\
f_{i}(t)=a_{i} t+b_{i} \\
p_{i}(t)=m_{i} \cos \left(\omega_{i} x\right)+n_{i} \sin \left(\omega_{i} x\right)+c_{i}
\end{array}\right.
$$

Where $f_{i}(t)$ represents the trend term at time ${ }^{i}$ of $Y_{i}(t), p_{i}(t)$ represents the cycle term at time tof $Y_{i}(t), a_{i}, b_{i}, m_{i}, n_{i}, c_{i}, \omega_{i}$ represent real-time rolling factors and parameters, which will be revised after getting the actual value at $i-1$ moment.

In order to realize real-time forecasting, the specific method is as follows.

1. Use these known values before moment to fit the time series model.

2. Utilize to predict the future 16 points of power value.

3. For the moment, use the obtained value at time and these known values to fit the new.

4. By such analogy, to correct the model in real time, making forecast results as accurate as possible.

Grey model. The most representative of gray modeling is GM modeling of time series [6]. In this paper, we choose the traditional gray model GM $(1,1)$.

Set up the wind power sequence: $p^{(0)}=\left(p^{(0)}(1), p^{(0)}(2), \cdots, p^{(0)}(n)\right)$, then do one accumulation to generate series and the mean number sequence: $p^{(1)}=\left(p^{(1)}(1), p^{(1)}(1)+p^{(0)}(2), \cdots, p^{(1)}(n-1)+p^{(0)}(n)\right)$ $z^{(1)}=\left(z^{(1)}(2), z^{(1)}(3), \cdots, z^{(1)}(n)\right)$, where $p^{(1)}(k)=\sum_{i=1}^{k} p^{(0)}(i), z^{(1)}(k)=0.5 p^{(1)}(k)+0.5 p^{(1)}(k-1)$.

The establishment of grey differential equation is

$$
p^{(0)}(k)+a z^{(1)}(k)=b, k=2,3, \cdots, n
$$

And albinism differential equation is

$$
\frac{d p^{(1)}}{d t}+a p^{(1)}(t)=b
$$

Note that $u=(a, b)^{T}, Y=\left(p^{(0)}(2), p^{(0)}(3), \cdots, p^{(0)}(n)\right)^{T}, B=\left[\begin{array}{cc}-z^{(1)}(2) & 1 \\ -z^{(1)}(3) & 1 \\ M & M \\ -z^{(1)}(n) & 1\end{array}\right]$, and by the least square method, to get $\hat{u}=(a, b)^{T}=\left(B^{T} B\right)^{-1} B^{T} Y$, so that $J(\hat{u})=(Y-B \hat{u})^{T}(Y-B \hat{u})$ obtains the minimum value.

Solving the equations, we can get:

$$
p^{(1)}(k+1)=\left(p^{(0)}(1)-\frac{b}{a}\right) e^{-a k}+\frac{b}{a}, k=1,2, \mathrm{~L}, n-1
$$

Among them, $p^{(0)}(k)$ represents the grey derivative, $a$ represents development coefficient, $\mathrm{b}$ represents gray ACTION, $B$ represents data matrix, $Y$ represents data vector, $u$ represents the parameter vector.

To achieve real-time forecasting, specific methods are as follows.

1. Turn 96 consecutive points into a $24 * 4$ matrix.

2. Use the above matrix to derive the next 4 values. 
3. When continue to seek the next 4 points, take the four points newly obtained as the last row of the matrix and delete the first line of the original matrix.

4. By such analogy, to correct the model in real time, making forecast results as accurate as possible.

Using the grey forecasting model to predict wind power value can find some "disaster point". In the given original data column, that below the specified value $\mathrm{q}$ is outlier. Take these ourliers as another sequence, in which every element is "disaster point " [7]. The disaster point can determine the location where error is larger.

Combination model. The combination forecasting method is proposed by Bates and Granger in 1969, and it's basic idea is to combine different forecasting methods and models via weighting [8]. For wind power forecast, based on the improved time series model and grey model, using fixed weight method, we establish a variance-covariance preferred combination forecasting model.

$$
\left\{\begin{array}{l}
P_{\Sigma}=\omega_{1} p_{T S}+\omega_{2} p_{G M} \\
\omega_{1}+\omega_{2}=1 \\
\omega_{1}=\frac{\operatorname{Var}\left(e_{G M}\right)}{\operatorname{Var}\left(e_{T S}\right)+\operatorname{Var}\left(e_{G M}\right)} \\
\omega_{2}=\frac{\operatorname{Var}\left(e_{T S}\right)}{\operatorname{Var}\left(e_{T S}\right)+\operatorname{Var}\left(e_{G M}\right)}
\end{array}\right.
$$

Among them, $p_{T S}$ and $p_{G M}$ represent unbiased prediction of improved time series model and gray model in turn, respectively, $\omega_{1}$ and $\omega_{2}$ are their weight.

The model diagram is shown in Fig.1.

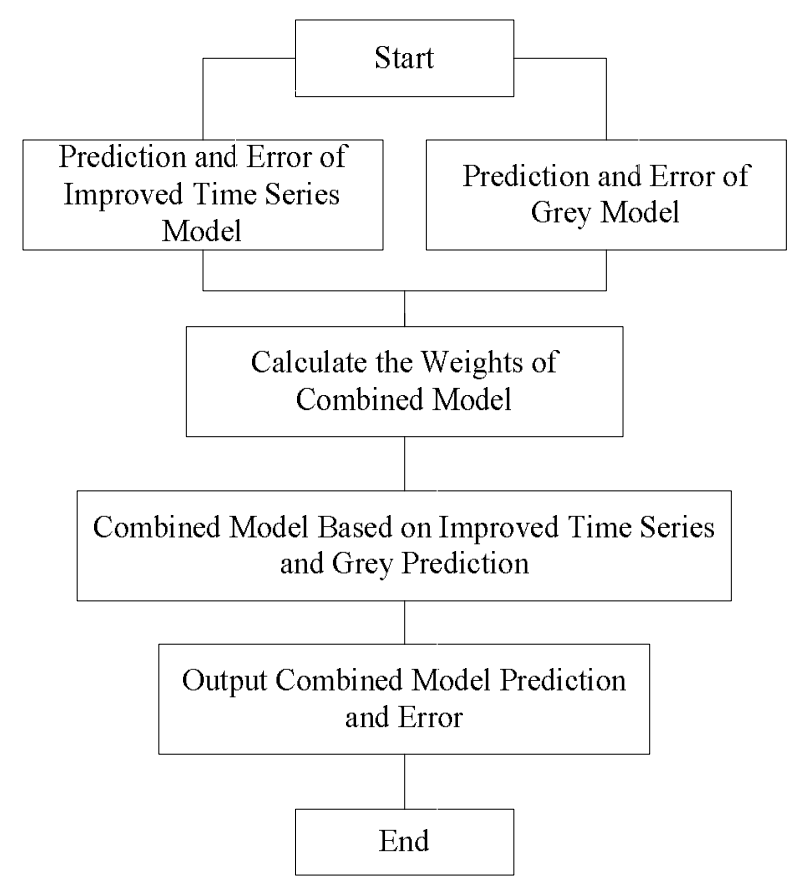

Fig.1 The flowchart of Combined Model

If single models errors are $e_{T S}$ and $e_{G M}$, the error of the combined model is $e=\omega_{1} e_{T S}+\omega_{2} e_{G M}$.

It can be proved that

$$
V \operatorname{ar}(e)_{\min } \leq V \operatorname{ar}\left(e_{T S}\right), V \operatorname{ar}(e)_{\min } \leq V \operatorname{ar}\left(e_{G M}\right) \text {, and } V \operatorname{ar}(e)_{\min } \leq \min \left(V \operatorname{ar}\left(e_{T S}\right), V \operatorname{ar}\left(e_{G M}\right)\right) \text {. }
$$

In other words, the perspective of mathematical analysis shows that the variance-covariance preferred combination forecasting method is better than the single model [9]. 
Model checking. In this paper, the accuracy rate and the qualified rate [10] are used as the wind farm power prediction assessment indicators.

The accuracy rate is

$$
\mathrm{r}_{1}=\left(1-\sqrt{\frac{1}{N} \sum_{k=1}^{N}\left(\frac{p_{M k}-p_{P k}}{C_{a p}}\right)^{2}}\right) \times 100 \%
$$

Among them, the actual average power of is $P_{M K}, P_{P K}$ represent the predicted average power, N represent the total time period of assessment day, and $C_{a p}$ represent the capacity of wind farm.

The qualified rate is

$$
r_{2}=\frac{1}{N} \sum_{k=1}^{N} B_{k} \times 100 \%
$$

Among them,

$$
\begin{aligned}
& \left(1-\frac{p_{M k}-p_{P k}}{C_{a p}}\right) \times 100 \% \geq 75 \%, B_{k}=1 \\
& \left(1-\frac{p_{M k}-p_{P k}}{C_{a p}}\right) \times 100 \%<75 \%, B_{k}=0 .
\end{aligned}
$$

\section{Example analysis}

Taking the measured data for one month of a wind farm as sample data and sampling interval is 15 min. Each wind unit has a rated output power of $850 \mathrm{~kW}$. The output power of four designated wind units $\mathrm{A}, \mathrm{B}, \mathrm{C}, \mathrm{D}$ is recorded as $\mathrm{P}_{\mathrm{A}}, \mathrm{P}_{\mathrm{B}}, \mathrm{P}_{\mathrm{C}}, \mathrm{P}_{\mathrm{D}}$, and the total output power of the generator is denoted as P.

Data correction. Models associated with time series can predict a wider range and has a high accuracy and simple form easy to master when the sequence is relatively stable. However, if the values of sequence are not steady, or the rise or fall in the sequence for some stage is too severe, the model reflects poor and can not give an accurate prediction value. Based on this, a method of data correction based on the picture of historical data to determine the range of time series is proposed, making a preliminary correction to sample data.

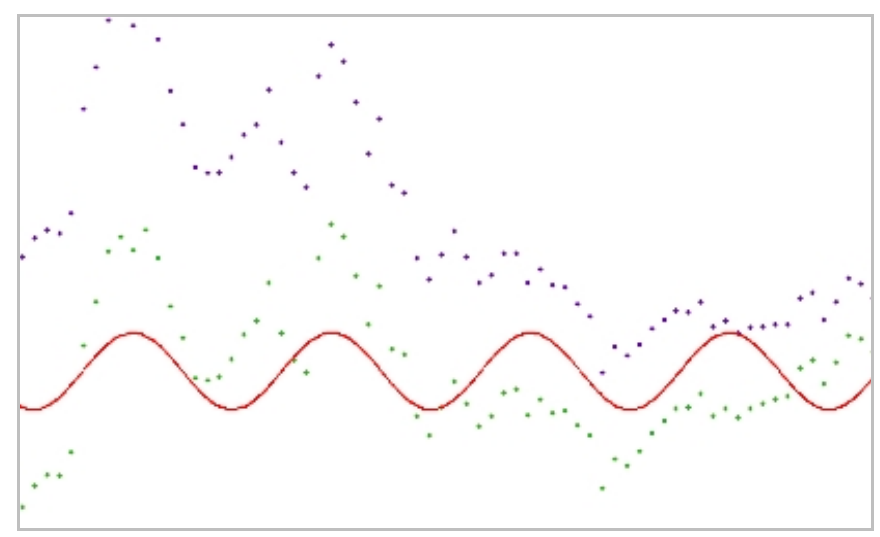

Fig.2 Sample diagrams

As shown in Fig. 2, the numerical gap between the first half and the second half is too large. When predicting the second half based on the first half, it will cause serious error. At this point, we can modify the range of fitting reference points with the help of graphics. Table 1 shows that the unrevised forecast 
value is not in conformity with the requirements. If such a situation occurs during the operation, be sure to redefine the scope of the time series, and then correct the model according to the rolling view.

Table 1 A comparison of before and after correction

\begin{tabular}{|c|c|c|}
\hline Primitive values & Preliminary revised value & Actual value \\
\hline-455.61 & 360.449 & 645.532 \\
\hline-603.08 & 358.384 & 1123.38 \\
\hline-701.67 & 425.336 & 1709.63 \\
\hline-739.58 & 525.759 & 1787.47 \\
\hline-717.99 & 596.435 & 1979.06 \\
\hline
\end{tabular}

Result comparison. Take $\mathrm{P}_{\mathrm{A}}$ for example, apply the above-mentioned single model and combined model to predict the future 16 point at the time of the specified point, the comparison between the results of the forecast and the actual value is shown in Fig. 3 below.

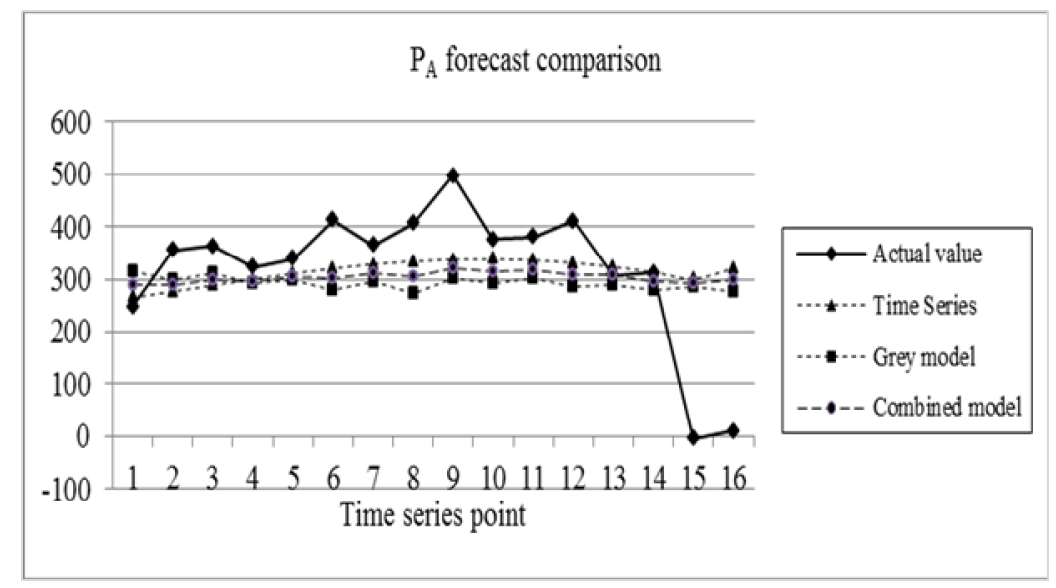

Fig. $3 \mathrm{P}_{\mathrm{A}}$ forecast comparison

1. By the image, the improved time series, grey model and combined model all have certain longitudinal error. But they also have a strong timeliness according to that the transverse errors is almost zero.

2. The fit at last two points is not optimistic. Through observation, at this stage the actual value plunge from 300 to 10 or even become negative, which we speculate this outcome may be caused by the stop operation of the unit.

Continue to calculate the accuracy rate and the qualified rate of the three models, as shown in fig. 4, fig. 5 .

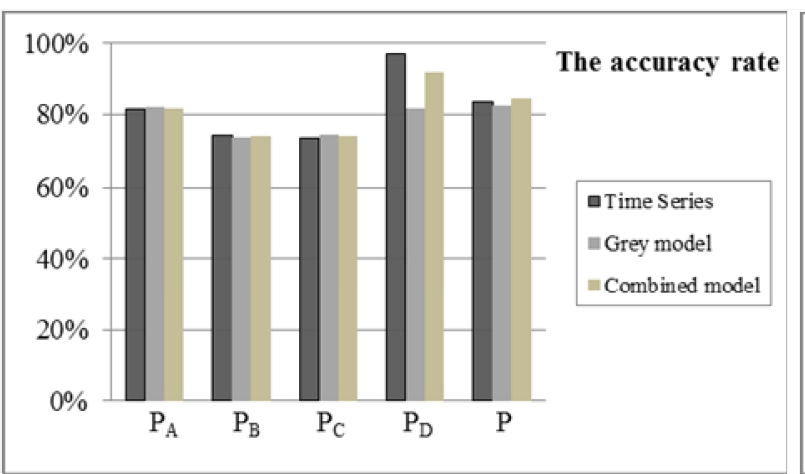

Fig.4 Forecast accuracy comparison

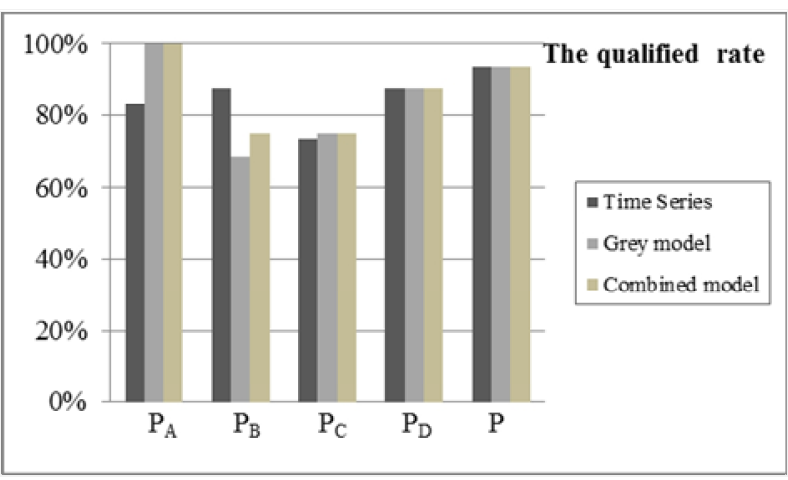

Fig.5 The qualified rate comparison

1. As can be seen from Fig. 4, the combined model has a relatively high average accuracy rate. With the increase in the number of units, the three models are in a normal distribution. 
2. From Fig. 5, it can be seen the qualified rate of combination model and grey prediction is higher, especially that of the combination model tends to be stable.

As a result, the variance-covariance preferred combination model modifies the prediction of large errors in the original model, whose accuracy is more stable and results are more reliable, overall improving the prediction accuracy. However, it also must be recognized that for the above model, main restricting factors to improve its precision is the nature of the sub model, if the sub model's accuracy generally continued to decline, the accuracy of optimized combination model is not too high.

\section{Conclusions}

1. Both single model and combined model can be applied to the real-time rolling forecast of wind power.

2. The algorithm of improved time series model is simple, the grey prediction of wind power can find some disaster points, and the variance-covariance combination model combines the advantages of both, overall improving the accuracy of the prediction.

3. It proposes a method of data correction based on the picture of historical data to determine the range of time series, which can modify sample data with the help of graphics.

4. In this paper, the combination forecasting model only use fixed weight method, optimizing weight coefficients can effectively improve the prediction accuracy.

\section{References}

[1] Tao Zhang, Xinyan Zhang, Weiqing Wang, Sichuan Electric Power Technology. 6 (2013) 16.(In Chinese)

[2] Liyang Liu, Junji Wu, Shaoliang Meng, Power System Protection and Control. 41 (2013) 65.(In Chinese)

[3] Lingling Li, Yahui Xu, Xiaoyue Tian, Transactions of China Electrotechnical Seciety. 29 (2014) 475-480.(In Chinese)

[4] Songlin Zhou, Meiqin Mao, Jianhui Su, Power System Technology. 35 (2011) 128-132.(In Chinese)

[5] Yi Luo, Feng Liu, Xiangjie Liu, Power System Protection and Control. 40 (2012) 47-53.(In Chinese)

[6] Dongxiao Niu, Bo Zhang, Lirong Zhang, East China Electric Power. 34 (2006) 8-11.(In Chinese)

[7] Wenwen Zhang, Systems Engineering. Supplements (1993) 362-368.(In Chinese)

[8] Jinwei Fu, Jin Ma, Yuxiao Zhou, East China Electric Power. 40 (2012) 889-893.(In Chinese)

[9] Dongxiao Niu, Shuhua Cao, Jianchang LuPower load forecasting technology and its application, China Electric Power Press, Beijing,2009.

[10] Miao Di, Minggang Wang. Science Technology and Engineering. 12 (2012) 7714-7717.(In Chinese) 